\title{
An Effective Iris Recognition System Using Fourier Descriptor And Principle Component Analysis
}

\author{
Ismail A. Ismail ${ }^{1}$, Mohammed A. Ramadan ${ }^{2}$, Talaat. El danf ${ }^{3}$, Ahmed H. Samak ${ }^{4}$
}

\begin{abstract}
Importance of biometric user identification is increasing every day. One of the most promising techniques is the one based on the human iris. In this work we propose a new method for iris recognition. The proposed system first Detect the iris region using Canny algorithm operator and Hough transform. Then the system normalizes the iris region and removes any noise. The final step in our system is the feature extraction using Fourier Descriptor and Principle Component Analysis (PCA). The result experimentation was carryout out using CASIA database. The experimental results have shown that the proposed system yields attractive performances and could be used for personal identification in an efficient and effective manner.
\end{abstract}

Index Terms - Iris recognition, Fourier Descriptor, Principle Component Analysis, personal verification

\section{INTRODUCTION}

Biometrics is one of the most important and reliable methods for computer-aided personal identification. It has a wide range of applications, in government programs such as national ID cards, use in visas and visa processing, and in the war against terrorism, as well as having personal applications in areas such as logical and physical access control[1,2]. Iris recognition is the most accurate personal identification biometric and it is this that accounts for its use in identity management in government departments requiring high security [3, 4]. The iris contains abundant textural information. This is often extracted in current recognition methods. Flom and Safir first proposed the concept of automated iris recognition in 1987, but the most representational iris recognition was proposed by Daugman $[3,4]$.

He used multi-scale quadrature wavelets to extract texture phase structure information of the iris to generate a 256-byte iris code and compared the iris code using the Hamming distance. Wildes [5], matched images using Laplacian pyramid multi resolution algorithms and a Fisher classifier. This approach, however, has proven to be computationally expensive and is suitable only for verification. Boles et al., extracted iris features using a 1-D wavelet transform at various resolution levels to calculate the zero crossing representation [6], but this method has been tested only on a small database. Sanchez-Avila et al. [7] further developed the iris representation method proposed by Boles et al. They made an attempt to match using different similarity measures.

In this work we present a novel iris feature extraction technique and the remainder of this paper is organized as follows. Section 2 gives an overview of the database used in the evaluation. Section 3 Iris Localization using canny algorithm operator and Hough transform. Section 4 and section 5 provide Iris Normalization and Noise Reduction. Features used in this wok discussed in section 6. Experimental Results and Conclusions discussed in section 7 and 8 .

\section{DAtABASE}

The Chinese Academy of Sciences-Institute of Automation (CASIA) eye image database [8] containing 756 grey scale eye images with 108 unique eyes or classes and seven different images of each unique eye have been used in the experimentation. Images from each class are taken from two sessions with one month interval between sessions.

The images were captured especially for iris recognition research using specialized digital optics developed by the National Laboratory of Pattern Recognition, China. The eye images are mainly from persons of Asian decent, whose eyes are characterized by irises that are densely pigmented, and with dark eyelashes.

\section{IRIS LOCALIZATION}

To separate iris from eye, it is necessary to get the inner boundary and the outer boundary of iris. There is a gray difference between the pupil and iris, find the turning point of gray curve from the histogram of iris, this is the boundary between pupil and iris, also is inner boundary. The outer boundary can be obtained by using Canny algorithm operator [9] and Hough transform. In the figure 1(b), the two white circles are the inner and outer boundary of iris.

\footnotetext{
Manuscript received February 15, 2009

1.Professor, Dean, College of Computers and informatics, Zagazig University, Egypt

2.Professor, Department of Mathematics, Faculty of Science, Menofia University, Egypt

3.lecturer, Department of Mathematics, Faculty of Science, Menofia University, Egypt

4.Ass. Lecturer, Department of Mathematics, Faculty of Science, Menofia University, Egypt.
} 

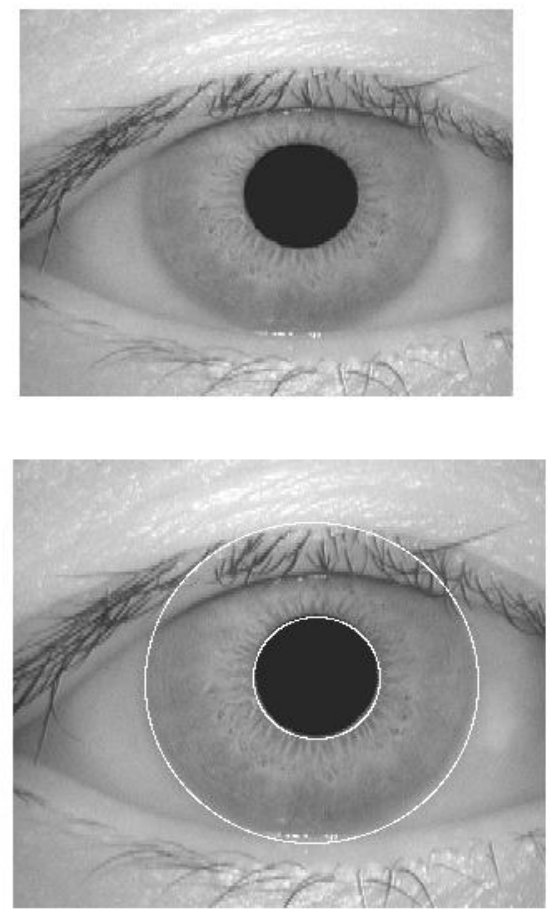

Fig.1 Iris images: (a) Original iris image (b) The iris image located

\section{IRIS NORMALIZATION}

The images of iris taken at different time or in different place have many differences, even in the same environment and from the same person, because elastic deformations of the pupil will affect the size of iris, the size of iris still has a large difference. To compensate the difference and improve the precision of matching, iris normalization is necessary. Because the inner and outer boundaries are circles approximately, doubly dimensionless projected polar coordinate system is a good method to normalize [10]. The result of normalization is shown in Fig.2

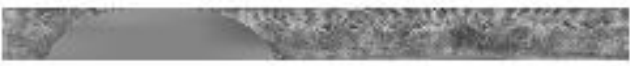

Fig. 2 Iris image after normalization

\section{NOISE REDUCTION}

The normalized image still is disturbed by the noise, such as the non-linear illumination and the disturber from the device. Standard noise reduction and isolated peak noise removal techniques, such as median-filtering and average filtering used to clear the noises and to make the iris textures more clear. The enhanced image is shown in Fig.3.

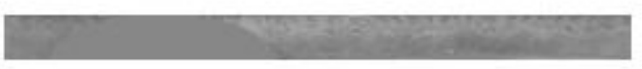

Fig.3 Iris image after enhancement and denoising

\section{IRIS FEATURES EXTRACTION}

Feature extraction of iris is the key of recognition. It is crucial to choose the suitable features. Considering the Fourier descriptor has many excellent attributes which is very suitable to extract the texture information..

\section{A. Fourier descriptor}

For any derived 1D iris function $u(t)$, its discrete Fourier transform is given by

$$
\begin{gathered}
a_{n}=\frac{1}{N} \sum_{t=0}^{N-1} u(t) \exp (-j 2 \Pi n t / N) \\
n=0,1, \ldots, N-1
\end{gathered}
$$

This results in a set of Fourier coefficients $\left\{a_{n}\right\}$, which is a representation of the iris region. Since image generated through rotation, translation and scaling (called similarity transform of a image) of a same image are similar images, a image representation should be invariant to these operations. The selection of different start point on the image boundary to derive $u(t)$ should not affect the representation. From Fourier theory, the general form for the Fourier coefficients of a contour generated by translation, rotation, scaling and change of start point is given by :

$$
a_{n}=\exp (j n t) \times \exp (j \phi) \times c \times a_{n}^{(0)} \quad n \neq 0
$$

where $a_{n}^{0}$ and $a_{n}$ are the Fourier coefficients of the original image and the similarity transformed image, respectively; $\exp (j n t), \exp (j \Phi)$ and $s$ are the terms due to change of starting point, rotation and scaling. Except the DC component $\left(a_{0}\right)$, all the other coefficients are not affected by translation.

Now considering the following expression

$$
\begin{aligned}
& b_{n}=\frac{a_{n}}{a_{0}}=\frac{\exp (j n t) \times \exp (j \phi) \times c \times a_{n}^{(0)}}{\exp (j t) \times \exp (j \phi) \times c \times a_{0}^{(0)}} \\
= & \frac{a_{n}^{(0)}}{a_{0}^{0}} \exp [j(n-1) t]=b_{n}^{(0)} \exp [j(n-1) t]
\end{aligned}
$$

where $b_{n}$ and $b_{n}^{0}$ are normalized Fourier coefficients of the derived image and the original image, respectively. The normalized coefficient of the derived image $b_{n}$ and that of the original image $b_{n}^{0}$ have only difference of $\exp [j(n-1) t]$. If we ignore the phase information and only use magnitude of the coefficients, then $\left|b_{n}\right|$ and $\left|b_{n}^{0}\right|$ are the same. In other words, $\left|b_{n}\right|$ is invariant to translation, rotation, scaling and change of start point. The set of magnitudes of the normalized Fourier coefficients of the iris image $\left\{\left|b_{n}\right|, 0<n \leq N\right\}$ can now be used as iris image descriptors, denoted as $\left\{F D_{n} 0<n \leq N\right\}$.

\section{B. PCA in iris recognition}

Let a iris image features extracted using Fourier descriptor is $\Gamma_{i}$ be $\mathrm{N}$ - element of one -dimensional image and suppose we have $\mathrm{M}$ images $(\mathrm{I}=1,2 . . \mathrm{M})$. A one-dimensional image column $\Gamma_{i}$ from the two-dimensional image (iris feature) is formed by scanning all elements of the two- dimensional image row by row and writing them to the column-vector. 
The main idea of PCA is to find the vector that beast account for distribution of these vectors within the entire image space. These vectors define the subspace of iris images features, called as " iris space " each vector of length $N$ elements, describes a $\mathrm{N}$ image, and is a linear combination of the original iris vectors.

Because these vectors are the eigenvectors of the covariance matrix corresponding to the original iris vectors, and because they are similar to iris in appearance, they are referred to as "eigeniris"

Let the training set of iris vectors be $\Gamma_{1}, \Gamma_{2}, \Gamma_{3}, \ldots \Gamma_{M}$ the averge vector of the set is defind by

$$
\Psi=\frac{1}{M} \sum_{n=1}^{M} \Gamma_{n}
$$

Each vectors differs from the average by the vector

$$
\Phi_{n}=\Gamma_{n}-\Psi
$$

Let the vectors $\mu_{k}$, and scalars $\lambda_{k}$ are the eigenvectors and eigenvalues, respectively, of the covariance matrix [1].

$$
\begin{gathered}
c=\frac{1}{M} \sum_{n=1}^{m} \Phi_{n} \Phi_{n}^{T}=A A^{T} \\
\text { Where } \\
A=\left[\Phi_{1}, \Phi_{2}, \Phi_{3} \ldots \Phi_{M}\right]
\end{gathered}
$$

In order to perform PCA, its necessary to find $\mu_{k}$ and $\lambda_{k}$. Because the dimensionality of N2 Of the matrix $\mathrm{C}$ is large even for a small images, and computation of eigenvectors using traditional method is complicated, dimensionality of matrix $\mathrm{C}$ is reduced using the decomposition describe in [8]. Found eigenvectors $\mu=\left(\mu_{1}, \mu_{2}, \ldots \mu_{N}\right)^{T}$ are normed and stored in decreasing order according to the corresponding eigenvalues. Then these vectors are transposed and arranged to form the row-vectors of transformation matrix $\omega_{n}$ (eigeniris)

\section{Using eigeniris to classify a iris image}

A new iris image feature $\Gamma$ is transformed into its eigeniris components (projected onto "iris space") by a simple operation [1]

$$
\omega_{n}=\mu_{n}(\Gamma-\Psi) \quad n=1,2 ., \ldots, M^{\prime}
$$

This describe a set of point-by-point image multiplications and summations. The weights from a vector $\Omega^{T}=\left[\omega_{1}, \omega_{2}, \ldots, \omega_{M^{\prime}}\right]$ that describe the contribution of each eigeniris in representing the iris image, treating the Eigen iris as a basis set for iris images.

The vector may thin used by a standard pattern recognition algorithm to find which of the number of predefined iris classes, if any, best describe the iris the next section discuses some distance measured can be used.

\section{Distance MEASURES}

Let $X, Y$ be eigeniris feature vectors of length $\mathrm{n}$. Then we can calculate the following distances between these feature vectors

(1) Minkowski distance ( $\mathrm{L}_{\mathrm{P}}$ matrices)

$$
d(X, Y)=L_{p}(X, y)=\left(\sum_{i=1}^{n}\left|x_{i}-y_{i}\right|\right)^{1 / P}
$$

(2) Manhattan distance $\left(\mathrm{L}_{1}\right.$ matrices, city block distance)

$$
d(X, Y)=L_{p=1}(X, y)=\sum_{i=1}^{n}\left|x_{i}-y_{i}\right|
$$

(3) Euclidean distance ( $\mathrm{L}_{2}$ matrices)

$$
d(X, Y)=L_{p=2}(X, y)=\sqrt{\sum_{i=1}^{2}\left(X_{i}-y_{i}\right)^{2}}
$$

(4) Angle - based distance

$$
\begin{aligned}
& d(X, Y)=-\cos (X, Y) \\
& \cos (X, Y)=\frac{\sum_{i=1}^{n} x_{i} y_{i}}{\sqrt{\sum_{i=1}^{n} x_{i}^{2} \sum_{i=1}^{n} y_{i}^{2}}} ;
\end{aligned}
$$

(5) Correlation coefficient- based distance

$$
\begin{aligned}
& d(X, Y)=-r(X, Y) \\
& r(X, Y)=\frac{n \sum_{i=1}^{n} x_{i} y_{i}-\sum_{i=1}^{n} x_{i} \sum_{i=1}^{n} y_{i}}{\sqrt{\left(n \sum_{i=1}^{n} x_{i}^{2}-\left(\sum_{i=1}^{n} x_{i}\right)^{2}\right)\left(n \sum_{i=1}^{n} y_{i}^{2}-\left(\sum_{i=1}^{n} y_{i}\right)^{2}\right)}} ;
\end{aligned}
$$

(6) Modified Manhattan distance

$$
d(X, Y)=\frac{\sum_{i=}^{n}\left|x_{i}-y_{i}\right|}{\sum_{i=1}^{n}\left|x_{i}\right| \sum_{i=1}^{n}\left|y_{i}\right|}
$$

(7) Modified SSE-based distance

$$
d(X, Y)=\frac{\sum_{i=1}^{n}\left(x_{i}-y_{i}\right)^{2}}{\sum_{i=1}^{n} x_{i}^{2} \sum_{i=1}^{n} y_{i}^{2}}
$$

\section{EXPERIMENTAL RESULTS}

This section reports some experimental results obtained using our method. In the following experiments, a total of 240 iris images (four iris images of each person is extracted). The experimental platform is the Intel core 2 duo $1.83 \mathrm{GHZ}$ processor, 1G RAM, Windows vista, and the software is 
Matlab 7.0.0.1. The method performance is evaluated using different distance measure as present in Table 1.

TABLE 1 : RESULTS OBTAINED USING VARIOUS DISTANCE MEASURE

\begin{tabular}{|c|c|}
\hline Distance measures & $\begin{array}{c}\text { Correct recognition } \\
\text { rate }\end{array}$ \\
\hline Minkowski distance & $93.75 \%$ \\
\hline Manhattan distance & $98.75 \%$ \\
\hline Euclidean distance & $95 \%$ \\
\hline Angle - based distance & $98.33 \%$ \\
\hline $\begin{array}{c}\text { Correlation coefficient- based } \\
\text { distance }\end{array}$ & $98.33 \%$ \\
\hline $\begin{array}{c}\text { Modified Manhattan distance } \\
\text { Modified SSE-based } \\
\text { distance }\end{array}$ & $99.583 \%$ \\
\hline
\end{tabular}

The methods proposed by Daugman [3], Sanchez-Avila et al. [7], Ma et al. [11], Tisse et al. [12] and Chen et al. [13] are the best known among existing schemes for iris recognition. Therefore, we have chosen to compare the performance of our proposed method against that of these ones as table 2 . From the results shown in Table 2, we can observe that Daugman's method [3] and our proposed yield the best performances, followed by Chen et al. [13], Ma et al. [11], Sanchez-Avila et al. [7] and Tisse et al. [12].

TABLE2 : COMPARISON OF METHODS (CORRECT RECOGNITION RATE)

\begin{tabular}{|c|c|}
\hline Methods & Correct recognition rate \\
\hline Daugman [3] & $99.90 \%$ \\
\hline Sanchez-Avila et al. [7] & $97.89 \%$ \\
\hline Ma et al. [11] & $98.98 \%$ \\
\hline Tisse et al. [12] & $89.37 \%$ \\
\hline Chen et al. [13] & $99.35 \%$ \\
\hline Proposed method & $\mathbf{1} 99.583 \%$ \\
\hline
\end{tabular}

\section{CONCLUSIONS}

In this work we present a novel iris feature extraction technique based on Fourier descriptor and principle component analysis and we obtained a series of results to show the performance of the proposed method. First we detect the iris region using canny algorithm operator and Hough transform. Then the system normalizes the iris region and removes any noise. The final step is the feature extraction. Different distance measured to evaluate the results and Modified Manhattan distance give the best results. All experimental results have demonstrated that the proposed method achieves high performance in both speed and accuracy.

\section{REFERENCES}

[1] S. Prabhakar, S. Pankanti, A.K. Jain, Biometrics recognition: security and privacy concerns, IEEE Security \& Privacy 1 (2) (2003) Pages:33-42.

[2] A. Jain, A. Ross, S. Prabhakar, An introduction to biometric recognition, IEEE Trans. Circuit Syst. Video Technol. 14 (1) (2004) Pages: 4-20.

[3] J.G. Daugman, High confidential visual recognition by test of statistical independence, IEEE Trans. Pattern Anal. Mach. Intell. 15 (11) (1993) Pages: 1148-1161.

[4] J.G. Daugman, The importance of being random: statistical principles of iris recognition, Pattern Recognition 36 (2003) Pages :279-291.

[5] R.P.Wildes, Iris recognition: an emerging biometric technology, Proc. IEEE 85 (1997) Pages :1348-1363.

[6] W.W. Boles, B. Boashash, A human identification technique using images of the iris and wavelet transform, IEEE Trans. Signal Process.46 (4) (1998) Pages :1185-1188.

[7] C. Sanchez-Avila, R. Sanchez-Reillo, Iris-based biometric recognition using dyadic wavelet transform, IEEE Aerosp. Electron. Syst. Mag.17 (2002) Pages: 3-6.

[8] Chinese Academy of Sciences-Institute of Automation, Database of Greyscale Eye Images, http://www.sinobiometrics.com Version 1.0, 2003.

[9] J. Canny, "A computational approach to edge detection, " IEEE Trans. Pattern Anal Machine Intell, vol. PAMI-8, 1986, Pages: 679-698,

[10] Jafar M. H. Ali and Aboul Ella Hassanien "An Iris Recognition System to Enhance E-security Environment Based on Wavelet Theory" Advanced Modeling and Optimization, vol.15, no.2, (2003) Pages: 93-104,

[11] Ma, L., Tan, T., \& Wang, Y. Iris recognition using circular symmetric filters. In Processing of the 16th international conference on pattern recognition Vol. 2, (2002). Pages: 414-417.

[12] Tisse, C.-L., Torres, L., \& Robert, M. Person identification based on iris patterns. In Proceedings of the 15 th international conference on vision interface (2002).

[13] Chen, C.-H., \& Chu, C. T. High performance iris recognition based on 1-D circular feature extraction Expert Systems with Applications (2009), doi:10.1016/j.eswa.2009.01.033 